\title{
Elastic electron-deuteron scattering and two-body current operators in the Light-Front Hamiltonian Dynamics
}

\section{Giovanni Salmè*}

Istituto Nazionale di Fisica Nucleare, Sezione di Roma, P.le A. Moro 2, I-00185 Rome, Italy

E-mail: salmeg@romal.infn.it

\section{Tobias Frederico}

Instituto Tecnológico de Aeronáutica, DCTA, 12.228-900, São José dos Campos, SP, Brazil

E-mail: tobiaseita.br

\section{J. Adnei Marinho}

Istituto Nazionale di Fisica Nucleare, Sezione di Roma, P.le A. Moro 2, I-00185 Rome, Italy

E-mail: admarinho@gmail.com

\section{Emanuele Pace}

Phys. Dep. "Tor Vergata” University and INFN Sezione di Tor Vergata, Via della Ricerca

Scientifica 1, I-00133 Rome, Italy

E-mail: emanuele.pace@roma2.infn.it

\begin{abstract}
The electromagnetic properties of the deuteron are investigated within a Light-Front Hamiltonian Dynamics framework, with a current operator that contains both one-body and two-body contributions. In this work, we are considering new two-body contributions, with a dynamical nature generated within a Yukawa model and a structure inspired by a recent analysis of the current operator, that acts on the three-dimensional valence component and fulfills the Ward-Takahashi identity. Preliminary results for the magnetic moment are shown.
\end{abstract}

Light Cone 2010: Relativistic Hadronic and Particle Physics

June 14-18, 2010

Valencia, Spain

\footnotetext{
* Speaker.
} 


\section{Introduction}

A careful description of the deuteron, retaining only a finite number of baryonic and mesonic degrees of freedom, could help to single out new, experimental signatures, related to the underlying degrees of freedom (see [1,2] for recent reviews of the QCD effects in the deuteron). In the present context, careful description means an approach that is able to fulfill general properties, like the extended Poincare covariance (the discrete symmetries are included), Hermiticity and current conservation [3].

Deuteron electromagnetic (em) observables offer a valuable play ground for testing theoretical ideas (see, e.g., [4]). In particular, the challenges for theorists appear very stimulating in view of the Th. Jefferson Lab. (TJLAB) upgrading to $12 \mathrm{GeV}$, that should open very intriguing scenarios, as described, e.g., in PAC-34 and PAC-35 Reports [5]. But, the list of issues to be coped with is long, if one would like to address non standard effects, e.g. like the 6-quark bag [1, 2, 4]. In our opinion (see, also [4]), efforts should be invested on the analysis of: i) the consistency between dynamics and operatorial structure of the current; ii) the strong interplay between different ingredients, e.g. between the dynamical content of the two-body currents and the nucleon form factors; iii) the two-photon exchange effects, that could affect the extraction of the em form factors (though recent estimates [6] assign them a minor role, $\sim 1 \%$ ); iv) further baryonic degrees of freedom, like isobar configurations, till now investigated only within a non relativistic approach [7].

Our aim is to include new dynamical two-body contributions into the em current, in a LightFront Hamiltonian Dynamics (LFHD) framework (see, e.g., [8] for a review of the Relativistic Hamiltonian Dynamics), for describing the deuteron em observables, still satisfying the extended Poincaré covariance. This work expands the investigation carried out in Refs. [9, 10], where a current operator containing the one-body term and a two-body contribution, needed for satisfying the Hermiticity, was considered. In the present approach, we add two-body terms with a dynamical nature, generated by the presence of an explicit one-pion exchange (see also [11]). These two-body currents are inspired by an exact analysis of the four-dimensional (4D) current corresponding to a Yukawa model in ladder approximation, where two fermions interact with a pseudoscalar, massive boson [12] (notice that, within such an approximation, there is no photon-boson coupling. The 4D current corresponding to the field theoretical model is projected onto the three-dimensional (3D) LF hyperplane, so that one obtains an operator, that i) acts on the 3D LF valence component of the interacting-system state and ii) automatically fulfills the Ward-Takahashi Identity (WTI). Moreover, in Ref. [12], it has been shown that one can properly truncate, in the Fock space, the LF current and still be able to satisfy the correspondingly truncated WTI. In particular, the Fock expansion is ordered in powers of the interaction. In order to improve the calculations of the em deuteron observables of Refs. [9, 10], in this work we consider the first-order LF current operator, obtained by applying the approach of Ref. [12] to an interacting Lagrangian $\mathscr{L}=-i g_{P S} \bar{\Psi} \gamma_{5} \vec{\tau} \Psi \cdot \vec{\phi}$, where $\Psi$ is the fermion field and $\vec{\phi}$ an isovector pseudoscalar boson field.

In Sec. 2, the choice of the reference frame and some generalities of our approach will be presented. In Sec. 3, the current adopted in our LFHD approach will be illustrated. In Sec. 4, the preliminary results, with only a part of the two-body dynamical contributions, will be shown for the deuteron magnetic moment. In Sec. 5 summary and perspectives will be presented. 


\section{Choosing the frame}

Our theoretical frame is the LF Hamiltonian Dynamics combined with the Bakamjian-Thomas construction [13] of the Poincaré generators for an interacting system. Within such a framework, as it is well known, a relativistic square mass operator of a two-body system can be immediately identified with the Schrödinger equation for that system [14]. This allows one to formally embed the standard non relativistic deuteron wave function into a fully Poincaré covariant description. The only particular care is the treatment of the spin part, where the Melosh rotation operators [15] are requested (see, e.g., [10] for details). Therefore, within the previous approach, one can rigorously fulfill the Poincaré covariance, for an interacting system with a finite number of degrees of freedom. Heuristically, one could say that the Relativistic Hamiltonian Dynamics with a Bakamjian-Thomas construction allow one to implement a description of an interacting system that falls between the non-relativistic quantum mechanics and local relativistic field theories. This simple view is further strengthened onto the LF hyperplane, since in this case a sharp and clean separation between the center of mass motion and the intrinsic one can be straightforwardly achieved. Let us also notice that the LF boosts are kinematical transformations and that the spectral condition $P^{+}=P^{0}+P_{z} \geq 0$ favors a description with a finite number of degrees of freedom.

The other ingredient for developing our description of the em deuteron form factors is the reference frame where we calculate the theoretical observables. If one had the complete and calculable theory at disposal, the choice of the frame should not represent an issue, given the full covariance,. Actually one is dealing with an approximate scheme, and therefore the choice of the reference frame, where one can more easily implement the constrains for preserving the general properties, becomes strategic. Following Ref. [3], one can show that in a Breit frame where the momentum transfer is longitudinal, i.e. $\vec{q}_{\perp}=0$, the symmetry of the physical process can be exploited for reducing the constraints imposed on the current operator by the extended Poincare covariance to a simple rotational covariance around the $\widehat{z} \equiv \widehat{q}$-axis. Then, in this frame, any operatorial dependence, fulfilling the rotational covariance around the $z$-axis, is allowed in the construction of a current operator, that, in particular, i) depends parametrically upon the CM momenta and ii) acts on the intrinsic variables. For instance, the matrix elements of the current operator can be approximated by the ones obtained from one-body Dirac and Pauli currents, and then, since the rotational covariance is safe, it turns out that the extended Poincaré covariance can be fulfilled by using the proper transformations (Lorentz boost, rotations, translations etc.). Moreover, in order to implement the Hermiticity, one has to add a term that contains the dynamical generator of the transverse rotations, obtaining necessarily a two-body contribution to the current. It should be pointed out that such a contribution must be distinct from the ones that we are going to discuss in what follows, since the last ones contain dynamical effects in a more explicit way.

\section{Projecting the em current onto the Light-Front hyperplane}

In this Section, a brief illustration of the results of Ref. [12], in particular the ones directly relevant for the present calculations, is given.

In Refs. [17, 18, 19, 12], by using the projection of the $4 \mathrm{D}$ physical quantities onto the 3D LF hyperplane (i.e. $x^{+}=x^{0}+x^{3}=0$ ), and the Quasi-Potential approach for the Transition Ma- 
trix [16], it has been established a formally exact, one-to-one correspondence between i) the 4D Bethe-Salpeter amplitude of an interacting system and the 3D LF valence component, ii) the matrix elements of the 4D em current and of the LF current, both fulfilling the proper Ward-Takahashi identity. The previous two ingredients allow one to keep separate the trivial propagation in the global time ( $\sum_{1, N} x_{i}^{+}$for a system with $N$ constituents), and to focus on the dependence upon the relative-time propagation, governed by the internal dynamics of the system. In particular, within this approach, the valence component is found to be the eigensolution of a 3D dynamical equation, with an effective 3D interaction that is exactly related to the $4 \mathrm{D}$ kernel of the BS equation. The exact correspondence between 3D and 4D quantities is accomplished through operators that contain the 4D interaction and cannot be determined in a simple way. This difficulty can be overcome in a workable way by developing a possible approximation scheme, for constructing solutions, based on the Fock expansion of the relevant quantities. If one truncates the Fock space, one can use the corresponding truncated Fock basis, in order to expand quantities, like the effective interaction and the current operator, onto the LF hyperplane. Clearly, by using a truncated space only the kinematical symmetries can be satisfied within a field theoretical approach (given the infinite number of degrees of freedom), but if one restricts to a Relativistic Hamiltonian Dynamics approach, where a finite number of degrees of freedom is taken into account, then one can adopt the truncated operators and recover the full Poincaré covariance. In particular, if the truncated current contains operators that satisfy the covariance around the $z$-axis, and furthermore one uses the valence wave functions, eigensolution of the properly truncated mass equation, in the evaluation of the matrix elements, then both the current conservation and the Poincare covariance can be implemented. It should be stressed that the truncated mass operator has to properly commute with the Poincaré generators, as requested by the Bakamjian-Thomas construction.

In the actual calculations, there is another relevant issue for the LF projection, that makes the application of the procedure to the fermionic case sharply different from the bosonic one. The Dirac propagator can be separated in an on-shell term and an instantaneous (in LF time !) propagation, viz

$$
i S(k)=\frac{k+m}{k^{2}-m^{2}+i \varepsilon}=\frac{k_{o n}+m}{k^{+}\left(k^{-}-k_{o n}^{-}+\frac{i \varepsilon}{k^{+}}\right)}+\frac{\gamma^{+}}{2 k^{+}}
$$

where $k_{o n}^{-}=\left(\left|\mathbf{k}_{\perp}\right|^{2}+m^{2}\right) / k^{+}$is the minus-component of $k_{o n}^{\mu}$, such that $k_{o n} \cdot k_{o n}=m^{2}$ and the second term is the instantaneous one, as shown by the Fourier transform in $k^{-}$and $\mathbf{k}_{\perp}$, i.e.

$$
\int d k^{-} d \mathbf{k}_{\perp} \exp \left[-i\left(k^{-} x^{+} / 2-i \mathbf{k}_{\perp} \cdot \mathbf{x}_{\perp}\right)\right]=(2 \pi)^{3} \delta\left(x^{+}\right) \delta\left(\mathbf{x}_{\perp}\right) .
$$

The instantaneous term has a great impact on the analysis of the matrix elements of the current operator, and it produces very peculiar operatorial structures in the many-body contribution to the LF current. It should be emphasized that this fact is related to our choice of the reference frame, where $q^{+} \neq 0$, since in this case the extraction of the em form factors involves matrix elements of both the plus and perp components of the current. In the frame with $q^{+} \neq 0$ the Poincare covariance can be demonstrated within a LFHD approach [3], and therefore the famous angular condition issue (see, e.g. [20]) does not any more plague the matrix elements.

In Ref. [12], the explicit expressions for many-body terms of the em LF current have been obtained, within a Yukawa model in ladder approximation for two fermions. In our approach for 

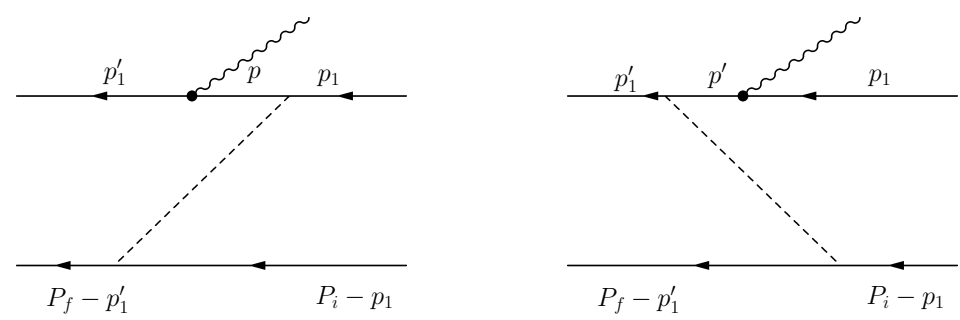

Interaction

terms
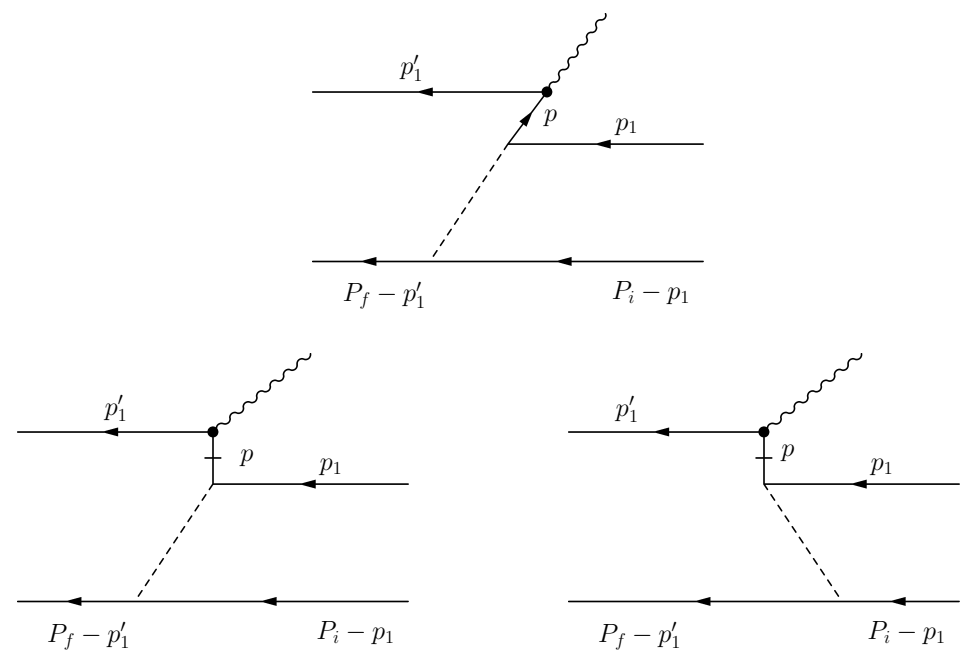

Pair term
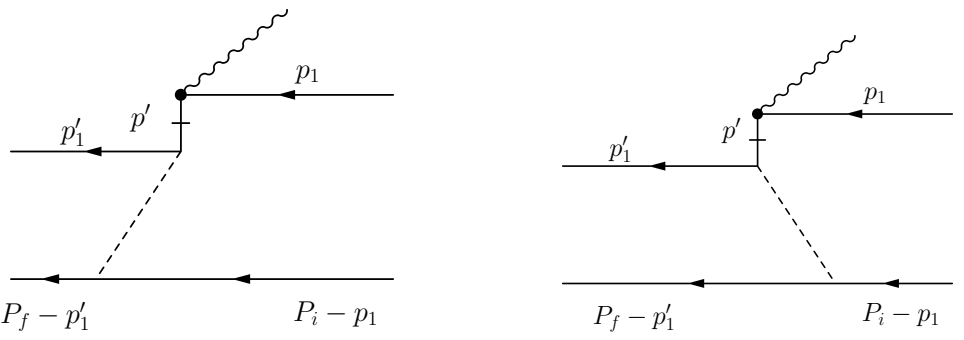

Instantaneous, in LF time, terms

Instantaneous, in LF time, terms

Figure 1: Diagrammatic analysis of the LF first-order em current, obtained within a Yukawa model in ladder approximation. In Ref. [12] one can find more details and explicit expressions.

evaluating the em deuteron observables, we take into account the current only up to the first-order in the interaction (see the next Section), as shown by the diagrammatic representation of Fig. 1. It is worth noting that one has at least three particles in flight (the LF-time flows from right to left).

\section{Preliminary results for the deuteron magnetic moment}

In a Breit frame where $\mathbf{q}_{\perp}=0$ and therefore $q^{+} \neq 0$, the matrix elements of the em current operator for an interacting system can be defined in terms of the LF current and the dynamical transverse component of the Bakamjian-Thomas rotation generator $\vec{S}_{\perp}$ as follows

$$
j^{\mu}\left(q \hat{e}_{z}\right)=\frac{\mathscr{J}^{\mu}\left(q \hat{e}_{z}\right)}{2}+L_{v}^{\mu}\left[r_{x}(-\pi)\right] e^{\imath \pi S_{x}} \frac{\mathscr{J}^{v}\left(q \hat{e}_{z}\right)^{*}}{2} e^{-\imath \pi S_{x}}
$$


where $L_{v}^{\mu}\left[r_{x}(-\pi)\right]$ is the $4 \mathrm{D}$ representation of the $\pi$-rotation around the $x$-axis, and generates a term necessary for implementing Hermiticity. In Eq. (4.1), the operator $\mathscr{J}^{\mu}\left(q \hat{e}_{z}\right)$ is given by

$$
\mathscr{J}^{\mu}\left(q \hat{e}_{z}\right)=j_{\text {one }}^{\mu}+j_{\text {two }}^{\mu}=\bar{\Pi}_{0} \mathscr{J}_{0}^{\mu} \Pi_{0}+\bar{\Pi}_{0}\left[V \Delta_{0} \mathscr{J}_{0}^{\mu}+\mathscr{J}_{0}^{\mu} \Delta_{0} V\right] \Pi_{0}
$$

where $\Pi_{0}$ is the so called free reverse LF projector of Ref. [12], that singles out the positiveenergy sector (modulo some kinematical factors)and $\Delta_{0} \equiv G_{\text {free }}-G_{\text {glob }}$, with $G_{\text {free }}$ the standard two-fermion free propagator and $G_{g l o b}$ an auxiliary Green's function, that takes into account the global-time propagation. The operator $V$ is the interaction, mediated by a pion (see also [11])

$$
V=i g^{2} \vec{\tau}_{1} \cdot \vec{\tau}_{2} \frac{\gamma_{1}^{5} \otimes \gamma_{2}^{5} \mathscr{F}^{2}\left[\left(\hat{p}_{2}-\hat{p}_{1}\right)^{2}\right]}{\left[\left(\hat{p}_{2}-\hat{p}_{1}\right)^{2}-m_{\pi}^{2}+i \varepsilon\right]}
$$

with $\mathscr{F}\left[\left(\hat{p}_{2}-\hat{p}_{1}\right)^{2}\right]$ the $4 \mathrm{D}$ vertex form factor counterpart of the nonrelativistic vertex function of Ref. [21]. The quantities with hats represent proper operators. In Eq. (4.2), the one-body contribution (see $[9,10]$ for details) is obtained from

$$
\mathscr{J}_{0}^{\mu}=\sum_{i=1,2}\left[J_{p i}^{\mu}(0) \frac{\left(1+\tau_{3 i}\right)}{2}+J_{n i}^{\mu}(0) \frac{\left(1-\tau_{3 i}\right)}{2}\right]
$$

where the free nucleon current is

$$
J_{N}^{\mu}=-F_{2 N}\left[\left(\hat{p}^{\prime}-\hat{p}\right)^{2}\right] \frac{\left(p^{\mu}+p^{\prime \mu}\right)}{2 M}+\gamma^{\mu}\left(F_{1 N}\left[\left(\hat{p}^{\prime}-\hat{p}\right)^{2}\right]+F_{2 N}\left[\left(\hat{p}^{\prime}-\hat{p}\right)^{2}\right]\right)
$$

with $F_{1 N}$ and $F_{2 N}$ the Dirac and Pauli form factors, respectively.

What about current conservation and charge normalization? In the chosen Breit frame, current conservation and charge normalization read respectively as follows

$$
\left\langle P_{f}, d\left|j^{+}\left(q \hat{e}_{z}\right)\right| d ; P_{i}\right\rangle=\left\langle P_{f}, d\left|j^{-}\left(q \hat{e}_{z}\right)\right| d ; P_{i}\right\rangle
$$

and

$$
\left.\left\langle P_{i}, d\right| j^{+}(0)\right)\left|d ; P_{i}\right\rangle=\left\langle P_{i}, d\left|\frac{1}{2}\left[\mathscr{J}^{+}(0)+\mathscr{J}^{-}(0)\right]\right| d ; P_{i}\right\rangle=e
$$

If WTI is fulfilled, then one obtains the current conservation, once matrix elements are taken between eigensolutions of the mass equation constructed from the proper Green' function. This is not the case in our phenomenological calculations, since we are adopting the deuteron wave functions corresponding to realistic interaction, like CD-Bonn [21] or AV18 [22]. But, in the elastic processes like the one we are considering, current conservation follows after implementing Hermiticity [3], given by (note the change of the $z$-axis)

$$
\left\langle P_{f}, d\left|j^{\mu}\left(q \hat{e}_{z}\right)\right| d ; P_{i}\right\rangle=\left\langle P_{i}, d\left|j^{\mu}\left(-q \hat{e}_{z}\right)\right| d ; P_{f}\right\rangle^{*}
$$

Notice that the charge normalization can be fulfilled if in Eq. (4.1) one defines

$$
\left\langle P_{i}, d\left|\mathscr{J}^{-}(0)\right| d ; P_{i}\right\rangle=\left\langle P_{i}, d\left|\mathscr{J}^{+}(0)\right| d ; P_{i}\right\rangle
$$

This leads to assume that such an equality holds for any momentum transfer. It should be pointed out that for evaluating the em observables only $j^{+}\left(q \hat{e}_{z}\right)$ and $j^{1(2)}\left(q \hat{e}_{z}\right)$ are relevant. 
Preliminary results for the magnetic moment of the deuteron have been obtained by retaining only the two-body interaction terms, corresponding to the diagrams depicted in the first line of Fig. 1, and with the explicit expressions given in Ref. [12] (modulo the isospin dependence and the pseudoscalar coupling). The nucleon form factors adopted in the calculations are the ones of Ref. [23], that are in nice agreement with the most recent measurements of the proton form factors (see, e.g., [25]) and represent a first microscopical interpretation of the possible zero in the ratio $\mu_{p} G_{E}^{p} / G_{M}^{p}$ for $Q^{2}>8(\mathrm{GeV} / \mathrm{c})^{2}$ in terms of interference between the valence and non valence component of the proton state. It is worth noting that the behavior of the nucleon form factors, in a wide kinematical region, enters in the evaluation of the static $\left(Q^{2} \rightarrow 0\right)$ em properties of the deuteron, according to the approach of Refs. [9, 10]. In Table 1, the preliminary values, for different deuteron wave functions, corresponding to three realistic NN interactions, are shown. In particular, the CD-Bonn [21], RSC93 [24] and AV18 [22] interactions have been used. The calculations corresponding to the one-body contribution with the Hermiticity term $[9,10]$ have been also shown. It should be mentioned that, within the last approximation scheme, the experimental quadrupole moment is fairly well described, with an underestimate of the order of $4 \%$.

\begin{tabular}{|c|c|c|c|c|}
\hline Interaction & $P_{D}$ & $\mu_{D}^{N R}$ & $\mu_{\text {one }}^{\text {LFD }}$ & $\mu_{1+2}^{\text {LFD }}$ \\
\hline CD-Bonn & 4.83 & 0.8523 & 0.8670 & $0.863 \pm 0.002$ \\
RSC93 & 5.70 & 0.8473 & 0.8637 & $0.861 \pm 0.002$ \\
Av18 & 5.76 & 0.8470 & 0.8635 & $0.860 \pm 0.002$ \\
\hline \multicolumn{5}{|c}{ Exp. 0.857406(1) }
\end{tabular}

The results appear encouraging. But, for a vanishing momentum transfer (see the analogous discussion for the one-body case in Refs. $[9,10])$, it is necessary an accurate study of the numerical convergence of the multifold integrals, that enter the calculations. From the charge normalization, one can obtain the probabilities of the valence and non valence components. At the present stage we have obtained $\operatorname{Prob}_{N V} \sim 0.01$.

\section{Summary \& Perspectives}

In this contribution, we have presented preliminary results for the magnetic moment of the deuteron, including two-body, dynamical contributions [12] to em current, within the LFHD approach proposed in Refs. $[3,9,10]$. The approach is fully Poincaré covariant.

The new two-body terms have been inspired by an exact analysis of a Yukawa model, in ladder approximation, for two interacting fermions, carried out in Ref. [12]. It turns out that, onto the LF hyperplane, one obtains a LF current fulfilling the Ward-Takahashi identity, at each order in the Fock expansion.

The systematic analysis of the deuteron em form factors is started, and it represents a non trivial task from the numerical point of view, given the many, multidimensional integrals to be performed with very high accuracy, in particular at low $Q^{2}$. First results obtained by using i) the deuteron wave functions corresponding to CD-Bonn, RSC93 and AV18 NN potentials and ii) the interaction contribution to the two-body current, as depicted in the first line of Fig. 1, appear consistent with the expectations, namely a very low probability for the component beyond the valence one, and a magnetic moment in fair agreement with the experimental values. Calculations of the quadrupole moments, more delicate from the numerical point of view, are in progress. 


\section{Acknowledgments}

This work was supported by the Brazilian agencies CNPq and FAPESP and by the Italian MUR.

\section{References}

[1] C. E. Carlson, J. R. Hiller, R. J. Holt, Annu. Rev. Nucl. Part. Sci. 47, 395 (1997).

[2] S.J. Brodsky, PoS(LHC07)002, and references quoted therein.

[3] F.M. Lev, E. Pace and G. Salmè , Nucl. Phys. A 641, 229 (1998).

[4] R. Gilman and F. Gross, J. Phys. G 28, R37 (2002), and references quoted therein.

[5] www.jlab.org/exp rog/PACpage/pac.html.

[6] Yu Bing Dong, Phys. Rev. C 80, 025208 (2009).

[7] A. Amghar, N. Aissat, B. Desplanques, Eur. Phys. J. A 1, 85 (1998).

[8] B. D. Keister and W. N. Polyzou, Adv. Nucl. Phys. 20, 225 (1991).

[9] F. Lev, E. Pace and G. Salmè, Phys. Rev. Lett. 83, 5250 (1999).

[10] F. Lev, E. Pace and G. Salmè, Phys. Rev. C 62, 064004 (2000).

[11] T. Frederico and R.W. Schulze, Phys. Rev. C 54, 2201 (1996).

[12] T. Frederico, J.A. Marinho, E. Pace, G. Salmè and P. Sauer, Phys. Rev. D 77, 116010 (2008).

[13] B. Bakamjian and L. H. Thomas, Phys. Rev. 92, 1300 (1953).

[14] F. Coester, S. C. Pieper, and F. J. D. Serduke, Phys. Rev. C 11, 1 (1975).

[15] H. Melosh, Phys. Rev. D 9, 1095 (1974).

[16] R. M. Woloshyn and A. D.Jackson, Nucl. Phys. B 64, 269 (1973).

[17] J. H. O. Sales, T. Frederico, B. V. Carlson, P. U. Sauer, Phys. Rev. C 61, 044003 (2000).

[18] J. H. O. Sales, T. Frederico, B. V. Carlson, P. U. Sauer, Phys. Rev. C 63, 064003 (2001).

[19] J. A. O. Marinho, T. Frederico, P. U. Sauer, Phys. Rev. D 76, 096001 (2007)

[20] L. L. Frankfurt, T. Frederico, and M. Strikman, Phys. Rev. C 48, 2182 (1993), and references quoted therein.

[21] R. Machleidt, Phys. Rev. C 63, 024001 (2001).

[22] R.B. Wiringa,V.G.J. Stoks, R. Schiavilla, Phys. Rev. C 51, 38 (1995).

[23] J.P.B.C. De Melo, T. Frederico, E. Pace, S. Pisano and G. Salmè, Phys. Lett. B 671, 153 (2009).

[24] V.G.J. Stoks, R.A.M. Klomp, C.P.F. Terheggen, J.J. de Swart, Phys. Rev. C 49, 2950 (1994).

[25] A.J.R. Puckett et al, Phys. Rev. Lett. 104, 242301 (2010). 